\title{
Anti-tumour Activity of Aqueous Wheat Grass Extracts against Chemically Induced Carcinogenesis
}

\author{
Caroline Wasonga ${ }^{1}$, Maureen Osoro ${ }^{2}$, \\ ${ }^{1,2}$ Department of Biochemistry, University of Nairobi. P.O. Box 30197-00100 Nairobi.
}

\begin{abstract}
Cancer is the leading cause of death globally and in 2015, cancer accounted for 8.8 million deaths worldwide. Liver cancer is the second most common type of cancer after lung cancer, causing many fatalities in both developed and developing countries. In attempting to manage the increasing cases of liver cancer and especially hepatocellular carcinoma, natural products like triticum aestivum (wheat grass) have been tested for their anti-oxidant and therapeutic properties. Treatment and prevention of hepatocellular carcinoma is still a challenge. This study aimed to investigate the effect of wheat grass on the progression of induced liver tumours in rats. Carcinogenesis was induced in experimental and positive group of rats. Development and progression of carcinogenesis was monitored by quantifying levels of alanine Transaminase, total sialic acid and by histological analysis. The results of the study showed that the levels of alanine transaminase and sialic acid consistently decreased throughout the experimental period on rats that were fed on wheat grass extracts compared to positive control. Histological analysis also showed protection of the hepatocytes from carcinogenesis development. Overall, the results from tumor markers and histological analysis, demonstrated that addition of wheat grass to the diet slowed down progression of carcinogenesis.
\end{abstract}

Keywords: Hepatocellular carcinoma, wheat grass extracts, carcinogenesis,

\section{Introduction}

Hepatocellular carcinoma (HCC) is the fifth most common malignancy (5\% of all cancer cases) and the third cause of cancer-associated mortality worldwide. It is also the most common primary hepatic malignancy with $80 \%$ of liver cancer cases being reported in adults and 35\% in children [1]. The main causes of liver disease leading to cirrhosis and/or HCC in Europe include hepatitis C infection in 60\% and hepatitis B infection in $15 \%$ of patients [2]. Other causes of HCC are carcinogens like aflatoxin and environmental pollutants, medications like estrogens, methyldopa and methotrexate. In addition, HCC is also caused by other life-style activities like alcohol abuse and long-term tobacco use [3]. Prevention and treatment of HCC has extensively been studied due to the gradual increase in the number of patients with this infection. One of the ways to manage $\mathrm{HCC}$ is by supplementation of diet using natural products like wheat grass. Wheatgrass is the young grass of common wheat plant, Triticum aestivum, belonging to the family Poaceae. It is commonly known for its high chlorophyll content which accounts for $70 \%$ of its chemical constituents. Wheatgrass contains 9 major components which include, 13 vitamins with antioxidants capability, many minerals and trace elements, muco-polysaccharides, abscisic acid, the antioxidant enzymes superoxide dismutase and cytochrome oxidase, which aid its fight against cancer [4]. Wheatgrass extract not only provides a unique source of nutrition, it also helps to regulate and reduce overactive elements in the immune system. This study aimed to determine the effect of wheat extracts on rats that have developed chemically induced carcinogenesis. This was monitored using tumors markers (sialic acid and alanine transaminase) and histological analysis.

\section{Materials and Methods}

Extraction of wheat grass active components: Fresh wheat grass was purchased from a fresh grocery store in Nairobi, Kenya. The wheat grass was cut into small pieces and dried in an incubator at $30^{\circ} \mathrm{C}$ overnight. It was then crushed to fine powder using a multi-grinder, and active components of wheat grass extracted using warm water. The extract was stored at $4^{\circ} \mathrm{C}$ until used.

Experimental Design: Twelve (12) rats were divided into three groups. Group One, consisting of three rats and not exposed to carcinogen, were fed on only mice pellets and water and were the negative control. Group Two, consisting of six rats, were fed on mice pellets and put on N-Nitrosodiethylamine for 13 weeks, and this was the positive control. Group Three, consisting of three rats, were fed on mice pellets, wheat grass extracts and exposed to N-Nitrosodiethylamine for 13 weeks and these were the experimental animals. The animals were bled on week $0,3,6,9$ and 12. At the end of week 13, two rats in each group were sacrificed under local anesthesia and their liver tissue fixed for histological examination. 
Tumour Induction: Induction of tumour was done by a modification of the method previously described by Ha et al., [5]. Rats were fed on $0.01 \%$ N-Nitrosodiethylamine (DENA) in drinking water for 13 weeks as the only drinking water to induce hepatic carcinogenesis.

Determination of Total Sialic Acid in Serum: Carcinogenesis progression was monitored by measuring sialic acid levels (experimental and controls), by a modification of a method previously described by Shambager et al., [6]. To each test tube, $0.01 \mathrm{ml}$ of serum, $0.9 \mathrm{ml}$ of distilled water and $0.2 \mathrm{ml}$ of Erhlich's reagent was added. Blank tubes containing $0.2 \mathrm{ml}$ of $\mathrm{HCl}: \mathrm{H}_{2} \mathrm{O}$ mixture in the ratio $1: 1$ was also prepared. Tubes were sealed with Parafilm, vortexed and incubated at $56{ }^{\circ} \mathrm{C}$ for 24 hours with occasional agitation. $3 \mathrm{ml}$ of $0.9 \mathrm{M}$ sodium chloride solution was added to each tube, vortexed and centrifuged at $1000 \mathrm{~g}$ for 10 minutes. Supernatant was aspirated and optical density read at $525 \mathrm{~nm}$ using a spectrophotometer.

Determination of Alanine Transaminase: Alanine transaminase in serum was assayed as previously described by Mohun and Cook [7]. $1 \mathrm{ml}$ of buffered substrate (alanine- $\alpha$-ketoglutarate pair) was put into a test tube and $0.2 \mathrm{ml}$ of serum added. The solution was mixed well and incubated for 30 minutes at $37^{\circ} \mathrm{C}$ in a water bath, $2.4 \mathrm{ml}$ dinitrophenyl hydrazine reagent added, mixed well and allowed to stand for 20 minutes at $37{ }^{\circ} \mathrm{C}$. Control tube with $1.0 \mathrm{ml}$ buffered substrate was incubated at $37^{\circ} \mathrm{C}$ for 30 minutes, serum added and $0.2 \mathrm{ml}$ of $1 \mathrm{mM} 2$, 4-dinitrophenylhydrazine reagent added, mixed and incubated for a further 20 minutes in the water bath. The tubes were removed from the water bath and $10 \mathrm{ml}$ of $0.4 \mathrm{M}$ sodium hydroxide added to the test and control tubes, mixed and allowed to stand for 10 minutes at room temperature, then the optical density read at $505 \mathrm{~nm}$ against a water blank.

Preparation and examination of histological slides: Two rats in each group were sacrificed under local anesthesia at the end of week 13 . Liver was cut into small pieces of approximately 1 cubic centimeter and fixed in bouin's reagent consisting of $75 \mathrm{ml}$-picric acid, $25 \mathrm{ml}$ formalin and $5 \mathrm{ml}$ acetic acid for 48 hours at room temperature. The tissues were transferred to the following solutions for 90 minutes each: $70 \%$ alcohol, $80 \%$ alcohol, two changes of absolute alcohol and two changes of xylene. Then the tissues were impregnated with pure molten paraffin wax at $50-60^{\circ} \mathrm{C}$ twice, and then mounted to labeled paper boats. The latter were floated on cold water to hasten solidification. The tissues were left at room temperature before sectioning using a microtome. The sections were then put on microscope slides and dried in an incubator at $37^{\circ} \mathrm{C}$ for 24 hours; stained with hematoxylin-eosin stains, then hydrated by immersing into two changes of xylene, absolute alcohol, $90 \%$ alcohol, $80 \%$ alcohol and $70 \%$ alcohol for 5 minutes each and finally hydrated with water, then stained with Hematoxylin for 30 minutes. Excess dye was washed off with excess water. The tissues were then differentiated by a quick deep in $200 \mathrm{ml}$ of $70 \%$ alcohol and $2 \mathrm{ml}$ hydrochloric acid alcohol solution and counterstained in Eosin stain for 3 minutes. Tissues were dehydrated by immersing in 50\% alcohol, $70 \%$ alcohol, $80 \%$ alcohol and $90 \%$ alcohol, then in two changes of absolute ethanol, xylene 1 and finally xylene 2, each done for 5 minutes. The mounted tissue was then covered with a cover slip after applying a thin layer of DPX mountant. Tissues were examined under a microscope (Ortholux, Leitz-Wetzler, Germany) for tumour incidence and photographs taken at X125 magnification.

\section{Results}

Potency of wheat grass extracts as anti-tumour agents was determined by feeding carcinogen exposed rats on pellets mixed with wheat grass extracts. In the animals fed on mice pellets only, the levels of sialic acid increased until week 3 , and then gradually decreased till week 12. In the animals exposed to carcinogen and normal diet, the total sialic acid levels increased consistently from weeks 0 to 12 . For those animals exposed to carcinogen and wheat grass extracts, the total sialic acid levels increased until week 6 and then decreased to week 12. These results are shown in Fig. 1.

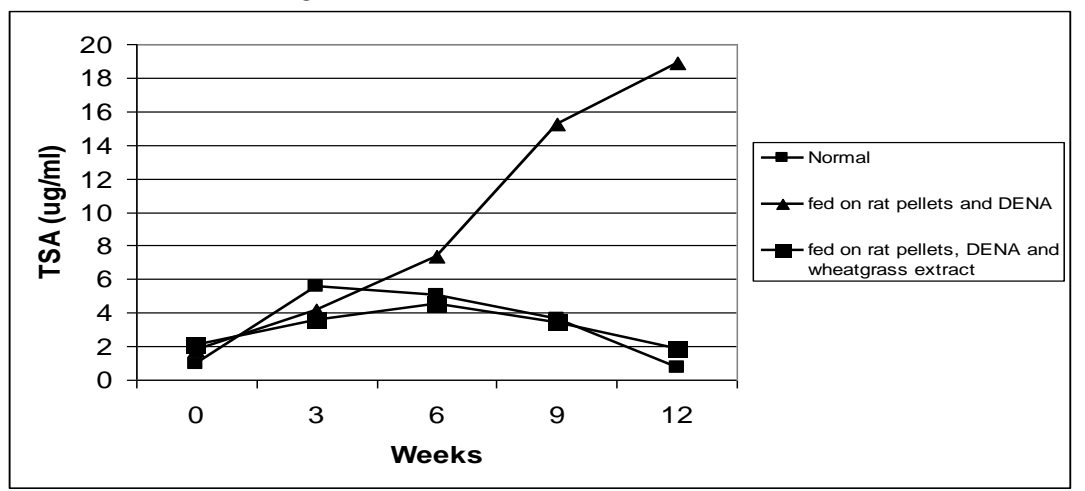

Figure 1: Trends of means of total sialic acid levels in the three groups of animals. Group 1 were fed on mice pellets only, group 2 were fed on carcinogen and mice pellets and group 3 were fed on carcinogen, mice pellets and wheat grass extracts. Samples were collected at the beginning of the experiment and at the end of weeks $3,6,9$ and 12 . 
Alanine transaminase levels, on the other hand, in the animals fed on mice pellets only remained constant throughout the 12-week period. In the animals exposed to carcinogen, the alanine transaminase levels increased consistently from onset of the experiment to 12 weeks and for those exposed to carcinogen and wheat grass extracts, the alanine transaminase levels increased until week 3 then decreased to week 12 as shown in Fig. 2.

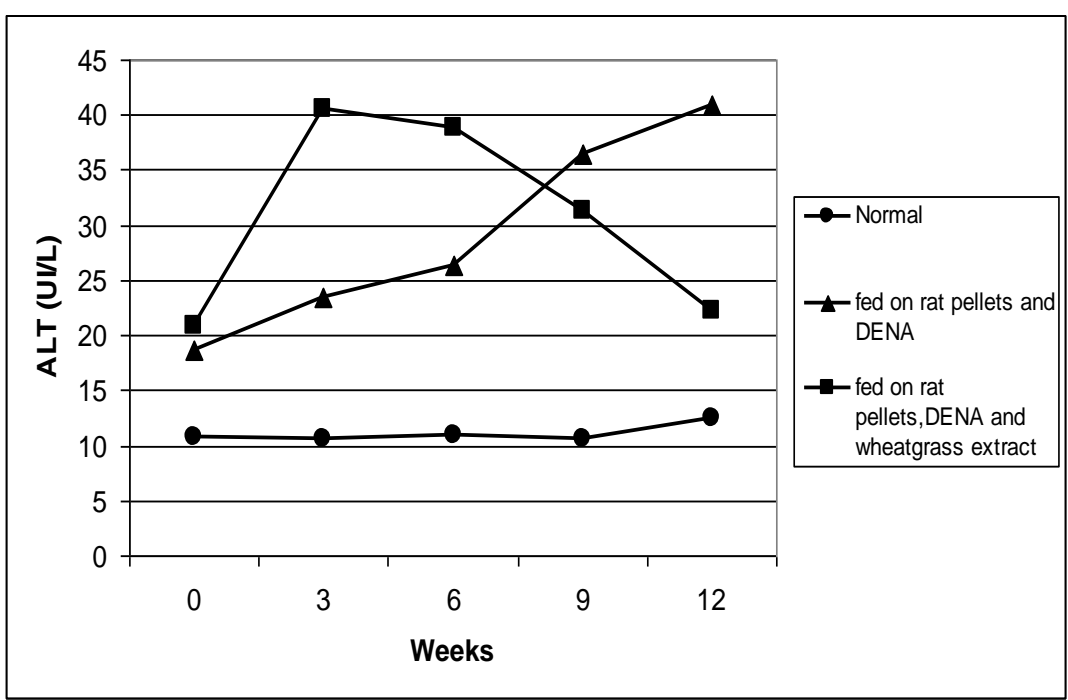

Figure 2: Trends of means of alanine transaminase levels in the three groups of rats. Group 1 were fed on mice pellets only, group 2 were fed on carcinogen and mice pellets and group 3 were fed on carcinogen, mice pellets and wheat grass extracts. Samples were collected at the beginning of the experiment and at the end of weeks 3 , 6,9 and 12.

Histological analysis demonstrated that by the end of week 13, the tissues of the rats fed on mice pellet only had prominent nucleoli and marginalized chromosomes characteristic of healthy cell as shown by Fig. 3a. On the contrary, histological appearance of liver tissue of the rat exposed to carcinogen and mice pellets only showed tissue with pale bodies and large nuclei as shown in Fig. 3b. The liver tissues from experimental animals had prominent nucleoli and marginalized chromosomes characteristic of healthy cells as shown in Fig. $3 \mathrm{c}$.

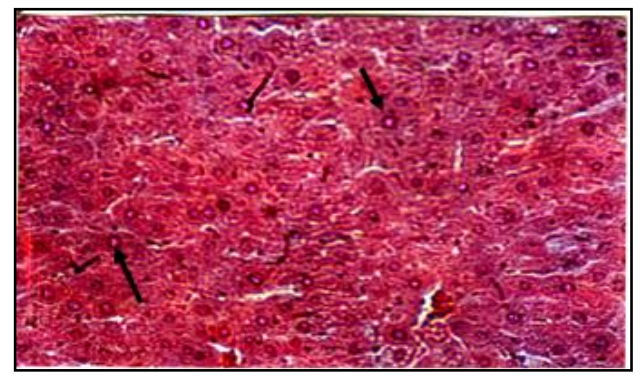

Figure 3a: Histological appearance of a healthy liver tissue that were not exposed to carcinogen but fed on pellets only. At the end of 13 weeks, liver tissue was harvested and processed for histological analysis. These tissues presented with prominent nucleoli and a marginalized chromosome representing a normal liver tissue (original magnification: x125).

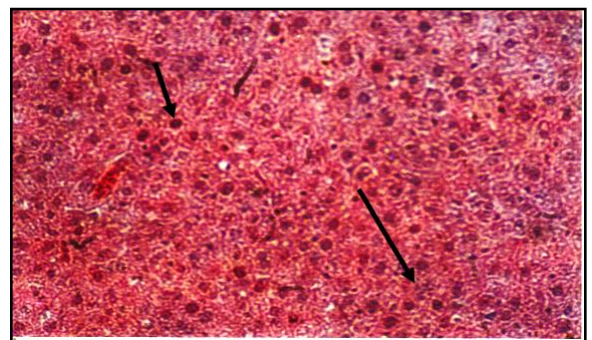

Figure 3b: Histological appearance of liver tissue that was exposed to carcinogen and fed on pellets only. At the end of 13 weeks, liver tissue was harvested and processed for histological analysis. These tissues presented with pale bodies and large nucleolus. (original magnification: x125). 


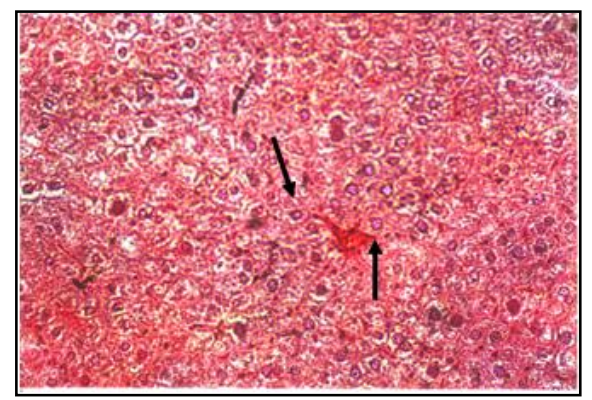

Figure 3c: Histological appearance of moderately differentiated mouse liver tissue that were exposed to carcinogen, fed on pellets and wheat grass extracts. At the end of 13 weeks, liver tissue was harvested and processed for histological analysis. These tissues presented with prominent nucleoli and a marginalized chromosome closely resembling a normal liver tissue (original magnification: x125).

\section{Discussion}

Cancer is a major cause of death in both developed and developing countries. In 2008, approximately 12.7 million cases of cancer were detected and 7.6 million people died from cancer worldwide [8]. Of these, $64 \%$ of the deaths occurred in the economically developing world. Hepatocellular cancer is becoming a disease of public health importance in developing countries because of the increasing cancer cases that have been reported. Some of the causes of hepatocellular carcinoma include environmental pollution due to urbanization, aging, adoption of cancer-associated lifestyle choices like smoking, drinking alcohol and physical inactivity [9 and 10]. Beyond monitoring lifestyle choices, we need to give importance to our diet. Some medicinal plants have been observed to confer anti-tumor properties due their anti-oxidant and therapeutic properties among other medicinal values.

To determine the effect of wheat extracts on rats that have developed chemically induced carcinogenesis, total sialic acid and alanine transaminase were quantified at every time point and histological analysis done. At the end of the 12 weeks experimental period, it was observed that the sialic acid and alanine transaminase levels consistently decreased in the animals that were exposed to carcinogen and fed on wheat grass extracts. This demonstrated that wheat grass extracts inhibited progression of tumours in the rats. This observation agrees with previous work done by Jain et al., [11], where liver enzymes used as biomarkers, showed hepatoprotective role of fresh wheatgrass juice in rats that were exposed to carcinogen. In another study, the wheat grass treatment prevented the increase of the liver enzymes, decreased oxidative stress and increased antioxidant levels with wheatgrass treatment [12].

The rats that were not exposed to carcinogen but fed on mice pellets only, presented with prominent nucleoli and marginalized chromosomes on histological examination of the tissues. This is characteristic of healthy liver cells. Liver cells absorbed the dye and were visualized as purple spots. For the rats that were fed on mice pellets and exposed to carcinogen, histology revealed dark spots and dye that was not absorbed. This is an indication that the cells present were either dead or damaged. The rats that were exposed to carcinogen, fed on mice pellets and wheat grass extracts showed tissue that had prominent nucleoli and marginalized chromosomes, which closely resembled those of healthy liver tissue. The tissue had pale bodies, large nuclei and relatively low decrease of chemosensitivity. Tumour markers and histological analysis in this study agrees with the theories put forth in other studies [13] that animals fed on wheat grass are healthier than those not fed on it, hence can be used synergistically with chemotherapy.

These results also agree with the theory by Bar-Sela et al., [14] that wheat grass extracts slows down progression of tumours. This study also corroborates findings of a previous study [15] where mushroom extracts delayed carcinogenesis progression in mice. This has been shown by the fact that rats exposed to carcinogen and wheat grass extracts in this study, resemble healthy rats as shown by levels of sialic acid and alanine transaminase levels and liver cell histology. In addition, a diet supplemented with wheat grass has been shown to delay carcinogenesis progression and extend the life of people with cancer, therefore, the use of wheatgrass as a supplement in terminally ill cancer patients should be encouraged [16 and 17]. Therefore, we recommend supplementation of wheat grass to our diets, to prevent or delay progression of carcinogenesis.

\section{Conclusion}

Deaths resulting from cancer continue to increase globally. Natural products like wheat grass extracts have shown promising results in delaying progression of hepatocellular carcinoma. In light of this study and previous reports reviewed herein and elsewhere, we recommend that wheat grass extracts be used to supplement conventional cancer therapy to manage HCC in well-controlled clinical trials. In these studies, fresh wheat grass should be administered for better results and caution should be exercised to avoid potential toxicity. 


\section{Acknowledgement}

We would like thank Mrs Katema and Mathew Kamau of Veterinary and human anatomy for technical support and appreciate the management of the Biochemistry department of the University of Nairobi for allowing us to access their facilities.

\section{References}

[1] F. X. Bosch, J. Ribes, R. Cléries, and M. Díaz, "Epidemiology of hepatocellular carcinoma," Clinics in Liver Disease, 9 (2), 2005, 191-211

[2] J. Sanyal, S. K. Yoon and R. Lencioni, The Etiology of Hepatocellular Carcinoma and Consequences for Treatment, The Oncologist, 15(4), 2010, 14-22

[3] H. B. El-Serag and K. L. Rudolph, "Hepatocellular carcinoma: epidemiology and molecular carcinogenesis," Gastroenterology, 132(7), 2007, 2557-2576

[4] R. Walters. The Alternative Cancer Therapy Book. New York, NY, USA: Avery Publishing Group; 1992

[5] W. S. Ha, C. K. Kim, S. H. Song and C. B. Kang, Study on mechanism of multi-step hepatotumorigenesis in rat: development of hepatotumorigenesis, Journal of Veterinary Sciences, 2(1), 2001, 53-58

[6] R. J. Shamberger, "Serum Sialic Acid in Normal and Cancer Patients," Journal of Clinical Chemistry \& Clinical Biochemistry, 22, 1984, 64-67.

[7] A. F. Mohun and I. J. Y. Cook, Simple Methods for Measuring Serum Levels of the Glutamic-oxalacetic and Glutamic-pyruvic Transaminases in Routine Laboratories, Journal of Clinical Pathology, 10(4), 1957, 394-399.

[8] A. Jemal, F. Bray, M. Melissa, J. Ferlay, E. Ward and D. Forman, Global Cancer Statistics, Cancer Journal for Clinicians, 61, 2011, 69-90

[9] M. J. Thun, J. O. DeLancey, M. M. Center, A. Jemal and E. M. Ward, The global burden of cancer: priorities for prevention, Carcinogenesis, 31(1), 2010, 100-110.

[10] A. Tfayli, S. Temraz, R. A. Mrad and Shamseddine, Breast Cancer in Low- and Middle-Income countries: An emerging and challenging epidemic. Journal of Oncology, 2010, http://dx.doi.org/10.1155/2010/490631

[11] G. Jain, A. Argal, A. K. Pathak, V. K. Singh and P. Kannojia, Hepatoprotective activity of wheatgrass juice, The pharmacist, 2(1), 2007, 29-30.

[12] J. K. Kamboj, S. V. Rana, R. P. Ola, D. K. Dhawan and K. Vahiphei, Wheatgrass and antioxidant levels in carbon tetrachloride induced hepatotoxicity in rats, Journal of Clinical and Experimental Hepatology, 1, 2011, S1

[13] G. Bar-Sela, M. Cohen, E. Ben-Arye and R. Epelbaum, The Medical Use of Wheatgrass: Review of the Gap Between Basic and Clinical Applications, Mini Review Medicinal Chemistry, 15(12), 2015, 1002-1010.

[14] G. Bar-Sela, M. Tsalic, G. Fried and H. Goldberg, Wheat Grass Juice May Improve Hematological Toxicity Related to Chemotherapy in Breast Cancer Patients: a pilot study, Nutrition and Cancer, 58(1), 2007, 43-48.

[15] O. C. Wasonga, A. S. Okoth, J. C. Mukuria and C. O. A Omwandho, Mushroom polysaccharide extracts delay progression of carcinogenesis in mice, Journal of Experimental Therapeutics and Oncology, 7 (2), 2008, 147-152

[16] P. Arya and M. Kumar, Chemoprevention by Triticum Aestivum of Mouse Skin Carcinogenesis Induced by DMBA and Croton Oil-Association with Oxidative Status, Asian Pacific Journal of Cancer Prevention,12, 2011, 143-148.

[17] S. Dey, R. Sarkar, P. Ghosh, R. Khatun, K. Ghorai, R. Choudhury, R. Ahmed, P. Gupta, S. Mukhopadhyay and A. Mukhopadhyay, Effect of Wheat Grass Juice in Supportive Care of Terminally Ill Cancer Patients - A Tertiary Cancer Centre Experience from India, Journal of Clinical Oncology, 24, 2006, S18. 